\title{
EFFECT OF BMI (BODY MASS INDEX) ON BMD (BONE MINERAL DENSITY) IN POSTMENOPAUSAL WOMEN
}

\author{
GHANI $\mathrm{T}^{1}$, PAUL SK ${ }^{2}$, BEGUM A ${ }^{3}$, NOORJAHAN ${ }^{4}$, SARKAR $\mathrm{M}^{5}$, HUSSAIN ${ }^{6}$, ANAR MGA ${ }^{7}$, \\ RANA MS ${ }^{8}$, HOSSAIN MS ${ }^{9}$
}

\begin{abstract}
Summary:
Menopause is commonly associated with rapid bone loss and this bone loss manifests as a significant decrease in bone mineral density (BMD). Body weight or body mass index is the most important factor which influences BMD. Aim of this study was to evaluate the association between $B M I$ and BMD in post menopausal women. Also to measure the correlation between age, duration of menopause, weight with BMD. This cross-sectional study was undertaken in the Department of Obstetrics and Gynaecology in Dhaka Medical College Hospital, Dhaka from January, 2012 to December, 2012. The study included women of 50 to 70 years who had menopause with three or more parity. Total 100 women were evaluated by history taking, physical examination and laboratory investigation (BMD). The results showed that there was significant positive correlation between BMI and BMD value of L1-4 and total femur (Pearson's coefficient was $+0.285, P<0.01$ and $+.350, P<.001)$. There was also significant positive correlation between weight and T- score of L1-4 $(r=+.482, P<.01)$ and Total femur $(r=+.513, P<.01)$. In addition, significant negative correlation was found between BMD with age and duration of menopause. It can be concluded that in postmenopausal women as the BMI decreases bone mineral density also decreases. So, adequate weight and BMI necessary for the prevention of osteoporosis.
\end{abstract}

J Dhaka Med Coll. 2019; 28(1) : 60-66

\section{Introduction :}

One of the most rapidly emerging global health problem in postmenopausal women is osteoporosis. Menopause is commonly associated with rapid bone loss, beginning 2-3 years before and continuing for up to 3-4 years after menopause. Good nutrition and healthy life style needed for higher BMD. But developing country like ours because of low socioeconomic condition most of the women suffers from malnutrition with low BMI. Low BMI is a important risk factors for occurrence of low BMD. Maintenance of adequate body mass is important for prevention of postmenopausal bone loss. Menopause usually occurs between ages of 45 and 52 years. Average age which is used to be 47 years in Britain and 51 years in
USA. ${ }^{1}$ Now more than $30 \%$ women are aged 50 years or above. Life expectancy is now 82 years in United Kingdom. In Bangladesh life expectancy is now 73.5 years for female. ${ }^{2}$ So, majority of women can therefore expect to live over a third of their lives in menopausal state but often this portion of life is not well enough due to body ache or low back pain, which is often ignored by the women and their family because of low socioeconomic condition. A better understanding of the relation between BMI and BMD provide an opportunity for early intervention and treatment to prevent fracture. The purpose of this study was to investigate the association between BMI and BMD and guide the clinician in the evaluation of fracture risk in postmenopausal women.

1. Dr. Tabassum Ghani, Associate Professor, Department of obstetrics and Gynaecology Dhaka Medical College, Dhaka.

2. Dr. Subinoy Krishna Paul, Associate Professor, Department of Surgery, Dhaka Medical College, Dhaka.

3. Dr. Afrina Begum, Associate Professor, Department of obstetrics and Gynaecology, Dhaka Medical College, Dhaka.

4. Dr. Noorjahan, Assistant Professor, Department of Obstetrics and Gynaecology, Dhaka Medical College, Dhaka.

5. Dr. Mandira Sarkar, Junior consultant (Gynae \& Obs) Upazilla health complex , Shibpur, Narsingdi

6. Dr. Taufiqua Hussain, Associate Professor, Department of obstetrics and Gynaecology, Dhaka Medical College, Dhaka.

7. Dr. Mosammat Gul - A- Anar, Senior Consultant (Gynae), 250 bed General Hospital, Tangail.

8. Dr. Md. Sohel Rana, OSD, DGHS, Dept. of Rheumatology, BSMMU, Dhaka

9. Dr. Md. Sazzad Hossain, Associate Professor, Department of Medicine, Shaheed Tajuddin Ahmed Medical College Gazipur Correspondence: Dr. Tabassum Ghani, Associate Professor (Gynae), Department of obstetrics and Gynaecology, Dhaka Medical College, Dhaka. Ph No: 01749135436, Email: tabassumghani@ymail.com

Received: 10 February $2019 \quad$ Revision: 02 March 2019

Accepted: 30 Mach 2019 
Body weight is positively associated with BMD from childhood through adulthood. Weight and body mass index are associated with low mineral density and fractures in older women. ${ }^{3}$ Body mass index or BMI is frequently suggested as one of the factors that indicates need for BMD screening for osteoporosis. Osteoporosis is a systemic skeletal disorder characterized by low bone mass and micro-architectural deterioration of bone tissue, with a consequent increase in fragility of bone susceptibility to risk of fracture. ${ }^{4}$ Osteoporotic fractures constitute a major public health problem. The main bone loss is in vertebra and Hip. Estrogen deficiency following natural or artificial menopause is thought to be main factor leading to bone loss. Osteoporosis is important because it causes morbidity and mortality. Osteoporotic fractures costs more than 10 billion dollars per year. By 2025, the annual fractures and costs are increase by $50 \%$ and 25 billion dollars respectively. ${ }^{5}$

BMI calculated by measuring height and weight of the patient. Height was measured by measuring tape with no shoe and weight also measured by weight machine. Formula of BMI - Weight in Kg divided by height in squared meter. According to Classification by WHO, BMI below $18.5 \mathrm{~kg} / \mathrm{m}^{2}$ is considered underweight; a BMI between 18.5 and $24.99 \mathrm{Kg} / \mathrm{m}^{2}$ is the ideal weight range; a BMI between 25 and 29.99 $\mathrm{kg} / \mathrm{m}^{2}$ is considered overweight; a BMI equal to or greater than $30 \mathrm{~kg} / \mathrm{m}^{2}$ is obese. (Giles, 2008). ${ }^{6}$

World Health Organization (WHO) used bone mineral density of Lumber spine and Hip measured by Dual X-ray Absorptiometry (DEXA). It has been shown to be very reliable and sensitive in diagnosis of osteoporosis. Results of bone mineral density can be reported as $\mathrm{T}$-score and $\mathrm{Z}$-score. $\mathrm{T}$ - score -It is the number of standard deviation above or below the mean Bone Mineral Density for gender matched young control. A negative value indicates that have thinner bones (low bone density) than an average 30 years old and a positive value indicates that have higher bone density than an average 30 year old. WHO in $1994{ }^{7}$ showed-
Normal: 2.5 to 1 below the young adult reference range $(2.5$ to -1$)$.

Low bone mass (Osteopenia): 1 to 2.5 SDs below the young adult reference range ( -1 to -2.5$)$.

Osteoporosis: 2.5 or more SDs below the young adult reference range (-2.5 or less).

Severe osteoporosis: 2.5 or more SDs below the young adult reference range (-2.5 or less) and the presence of one of bone fractures.

\section{Z-Score}

BMD value compared to other people of same age, sex and race. This is called $Z$-score. A low $Z$-Score (below -2.0) is a warning sign that have less bone mass than expected for someone of same age.

A negative value indicates that have thinner bones (lower bone density) and weaker bones than the average of people of same group. The more negative value is the less bone density have compared with others in same group.

A positive value indicates that have higher bone density and stronger bones than the average of people of in same group (Khan, 2004). ${ }^{8}$

A better understanding of this relation may provide opportunity to prevent and treatment of osteoporosis and thus prevent fracture.

\section{Aims and objective:}

To evaluate the association of BMI with BMD in postmenopausal women.

\section{Materials and Methods :}

This Cross-sectional study was carried out from January, 2012 to December, 2012 in the Department of Obstetrics and Gynaecology, $\mathrm{DMCH}$. The study population consisted of patients of 50 to 70 years who had natural menopause and cases were selected by purposive sampling. After fulfillment of eligibility criteria only 100 subjects were enrolled for the study. All postmenopausal women of 50-70 years of age who had undergone natural menopause with parity 3 or more included and patient had menopause before 50 years, patient had surgical menopause, chronic medical disorder like chronic renal disease, hypo or hyperthyroidism, Medication known to affect the bone such as patient on current 
therapy on- anticoagulant, anticonvulsant, corticosteroid, Hormone Replacement therapy were excluded from this study. Prior to commencement of this study the research protocol was approved by the ethical committee. The Aims and objectives of this study, it's procedure and benefit were explained to the patients in easily understandable local language. Informed consent was taken from each patient. It was assured that all information and records would be kept confidential and the procedure will be helpful for both the investigator and patients in making rational approach of the case management. Demographic variables were age (years), Parity, menopausal age and clinical variables were Height, Weight, BMI. Age was recorded in nearest full years stated by subject and Parity was recorded number of viable births reported by women. Height was measured by measuring scale and Weight was measured by weight machine. BMI or Body mass index was calculated from body weight in $\mathrm{Kg}$ divided by height in meters squared. Bone Mineral Density (BMD) expressed in $\mathrm{Gm} / \mathrm{cm}^{2}$. For the purpose of the study, Bone mineral density was be measured by Lunar Prodigy Advance DXA system (Analysis version : 12.30) at the lumber spine at the level of L1 to L4 and total femur by the technician of BMD laboratory. The measured BMD was automatically displayed. Data were processed and analyzed using SPSS (Statistical package for social Sciences) version 13.0. The test statistics used to analyzed the data were $\mathrm{F}$ test (ANOVA), Pearson's correlation test. For all analytical tests, the level of significance was set at 0.05 and .01 level was considered significant. Summarized data were presented in the form of tables and diagram.

\section{Results}

Out of all patients maximum 33 within 56 to 60 years of age range followed by 23 were within 61 to 65 and 66 to 70 years and only 21 were within 50-55 years range. Mean age of patients was 60.66 years with a standard deviation of \pm 6.25 years.

Parity were divided into three groups. 64 patients were in 3-5 para, 30 patients were in 6-8 para and 9 patients were 6 para. 37 had menopause over 11-15 years, 23 women had menopause of 1-5 years, 15 women had 6-10 years, 7 women had 16-20 years $\&$ over 20 years of menopause in 18 women. Mean age of menopause was $12.32 \pm 6.9$ years. Maximum (52\%) had normal weight, $24 \%$ had over weight, $16 \%$ were obese and only $8 \%$ were underweight. There was negative relationship of age of the patients with BMI. Mean age of the patients was $60.66 \pm 6.6$ and mean BMI was 24.55 \pm 4.7. Pearson's correlation coefficient was done. Here $r=-0.85$ and $p=0.401$, which was not significant. So, there was negative relation of age with BMI but not significant.

Among 100 patients, mean height and weight was about $149 \mathrm{~cm}$ and $57 \mathrm{~kg}$. Mean BMD value of L1-4 and Total femur was 0.905 and 0.822 $\mathrm{gm} / \mathrm{cm}^{\mathrm{m}}$ respectively. Eighty Three percent patient had Low BMD and 17\% patients had normal BMD whereas their mean T- score was -2.29 for the L12-4 and -1.46 for the total femur. Mean Z- score of L1-4 was -7.7 and Total femur was -2.88 . Bone mineral density was more in L1-4 than total femur.

Among 100 patients average weight of the patients was $57.19 \pm 10.13 \mathrm{~kg}$. T-score of L1-4 and Total Femur were -2.22(SD =1.4) and - 1.4 $(\mathrm{SD}=.13)$. Depending on $\mathrm{T}$ - score about $35 \%$ had normal Bone density, 48\% had low bone density and $17 \%$ had osteoporosis. There was positive correlation between weight and T-score of L1-4 and total Femur and it was statistically significant. $(\mathrm{r}=+.482, \mathrm{r}=+.513, \mathrm{P}<.01)$.

\section{Table-I}

Correlation of weight and T- score of L1-4 and Total Femur

\begin{tabular}{ccc}
\hline Weight & T-score of L1-4 & T- score Total Femur \\
\hline r value & $+.482^{* *}$ & $+.513^{* *}$ \\
P Value & .000 & .000 \\
\hline
\end{tabular}

** Pearson's correlation Significant at 0.01 Level.

Different variable were related with each other by positive or negative correlation. Following table showed that there was significant negative correlation between age and duration of menopause with BMD of L1-4 and Total femur. 
There was significant positive correlation of BMI with BMD value of L1-4 and Total femur.

Table -II

Distribution of study subjects by age, duration of menopause, BMI with BMD of L1-4 and Total femur

\begin{tabular}{lccc}
\hline & $\begin{array}{c}\text { BMD } \\
\text { L1-4 } \\
\text { r value }\end{array}$ & $\begin{array}{c}\text { BMD Total } \\
\text { Femur } \\
\text { r value }\end{array}$ & $\begin{array}{c}\text { P } \\
\text { value }\end{array}$ \\
\hline Age & -.212 & -.354 & $.034 / .014^{*}$ \\
Parity & -.224 & -.266 & $.021 / .009^{* *}$ \\
Duration of & -.258 & -.264 & $.010 / .008^{*}$ \\
Menopause & & & \\
BMI & +.285 & +.250 & $.004 / .000^{* *}$ \\
\hline
\end{tabular}

*Significant at $\mathrm{P}<.05$ Level.

** Significant at $<.01$ level.

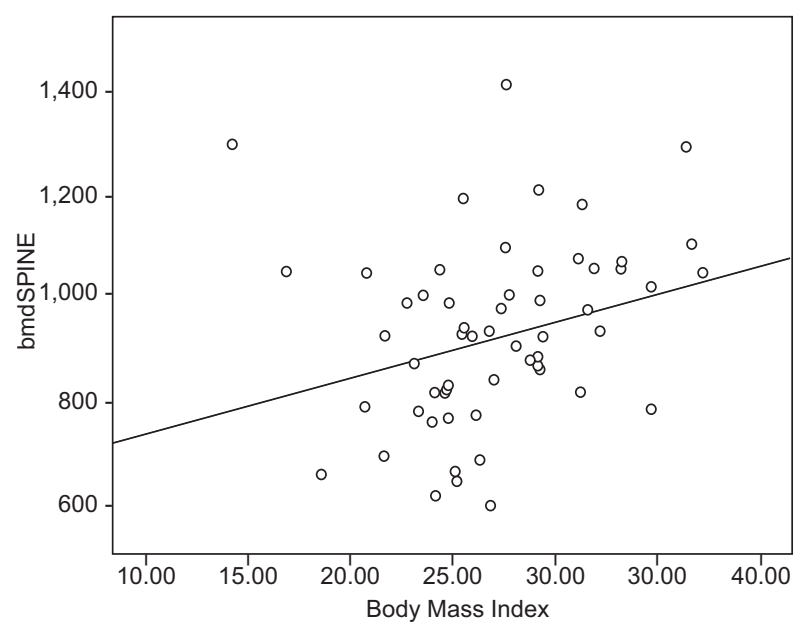

Fig.-1 : Correlation of BMD of L1-4 and BMI

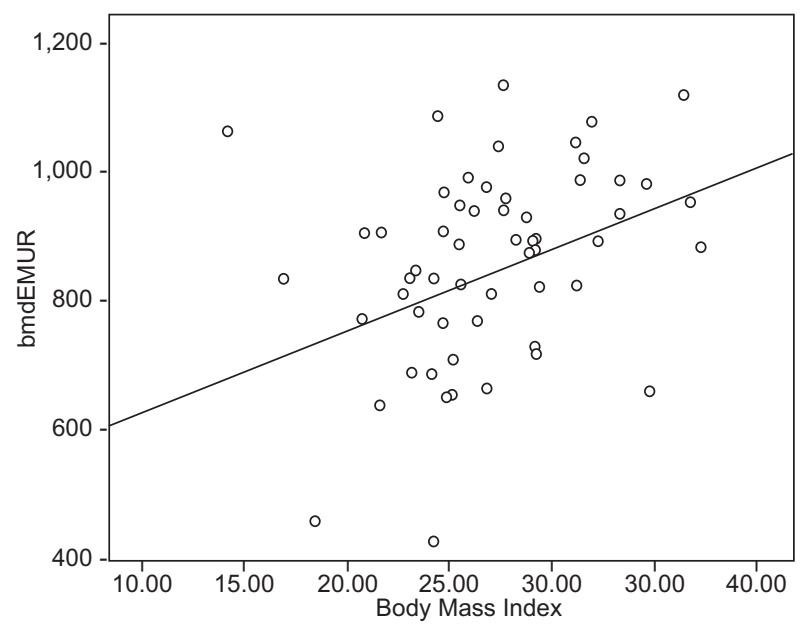

Fig.-2: Correlation between BMI with BMD Femur

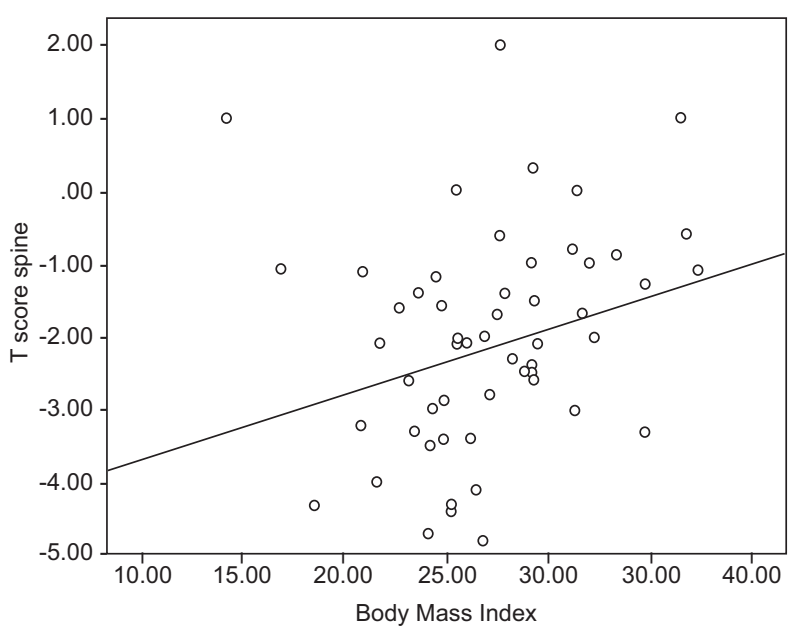

Fig.-3: Correlation of BMI with T-score L1-4.

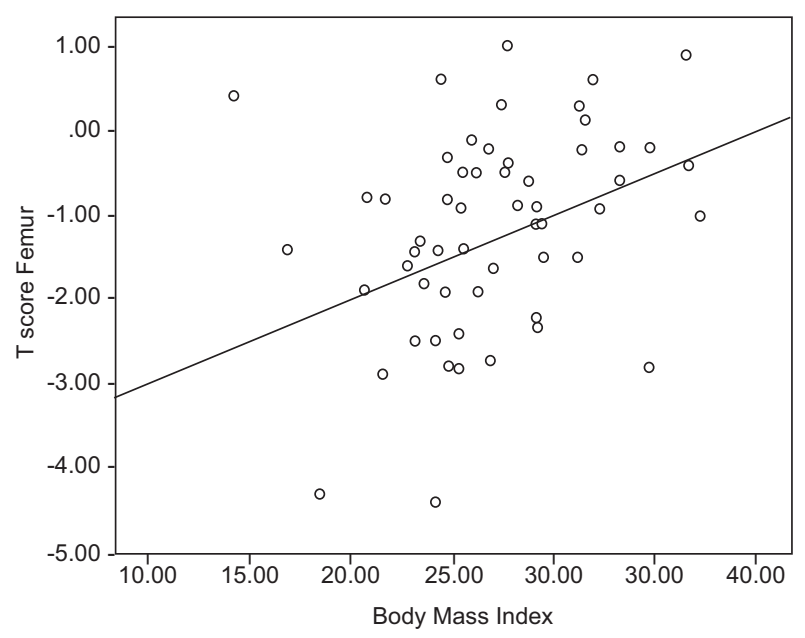

Fig.-4 : Correlation of BMI with T-score of Total Femur

\section{Discussion}

Osteoporosis is a serious and frequent disease that occurs worldwide. The most important risk factor for osteoporosis is estrogen deficiency, and the increasing life expectancy of women indicates that the problems of osteoporosis will be overwhelming in the years to come.

Thinness is a major risk factor for low bone mineral density (BMD). Thus thin, otherwise healthy women have a lower BMD and will lose bone faster than heavier women. Impact of thinness on risk of osteoporosis development is of particular interest in developing countries, because of the high incidence of starvation which leads to malnutrition. Other risk factors are age, duration of menopause, low physical activity and smoking. 
Among 100 postmenopausal women mean age was 60 years with SD of \pm 6.25 years. Differences in Body mass index among menopausal women was observed in this study. Majority women had BMI within normal range in my study which was $52 \%, 24 \%$ were overweight, $16 \%$ were obese and only $8 \%$ women were underweight. Mean $( \pm \mathrm{SD}) \mathrm{BMI}$ was 24.55 ( $\mathrm{SD}=4.7)$. In a study done by Masazumi (1996) ${ }^{9}$ showed that natural menopause did not exert a significant effect on BMI. In my study no significant relation was found between BMI and age which was similar with that study.

Paulo G. Pedreira $(2011)^{10}$ done a case control study with postmenopausal women showed their mean weight and height were $59.1 \mathrm{~kg}$ $(\mathrm{SD}=8.3)$ and $156.6 \mathrm{~cm} \quad(\mathrm{SD}=4.4)$, respectively, and their mean body mass index (BMI) was 24.1 (SD = 3.1). In our study mean weight and height were $7.19 \pm 10.93 \mathrm{~kg}$ and 149 $\pm 6.73 \mathrm{~cm}$. Their study showed mean calculated overall L1-4 BMD value was $1.02 \mathrm{gm} / \mathrm{cm}$ $(\mathrm{SD}=0.17)$ and mean $\mathrm{T}$-score and $\mathrm{Z}$-score were$0.85(\mathrm{SD}=1.42)$ and $-0.28(\mathrm{SD}=1.22)$ and in our study mean calculated L1-4 BMD value was $0.905 \mathrm{gm} / \mathrm{cm}(\mathrm{SD}=1.76)$ and Total Femur was $.822 \mathrm{gm} / \mathrm{cm}(\mathrm{SD}=1.7)$. Mean calculated $\mathrm{T}$ and $Z$ score were $-0.82(\mathrm{SD}=1.72)$ and -0.77 $(\mathrm{SD}=1.25)$ respectively. Their values were more or less similar to our study. Above study showed that age, postmenopausal length and BMI were all significantly correlated with BMD. In case of age and postmenopausal length, the correlations were negative, suggesting that BMD decreased as these women aged, or as the time since their menopause increased. Interestingly, BMI was positively correlated with BMD indicating that women with higher BMI in this population also had higher BMD which was in consistent with our study.

There was a positive correlation between BMI and BMD / T- scores, indicating that high BMI would be protective in maintaining higher $\mathrm{BMD}$, these findings could be specific to M. J Rapoport (2003). ${ }^{11}$ Most women in the current study were of normal weight $(43.8 \%$ with BMI between 18.5 and $23, n=29), 25.8 \%$ were slightly overweight (BMI between 25 and $30, n=17$ ) and only 3 had a BMI over 30 . Moreover, there was a trend in BMI values decreasing from the normal, to the lower BMD group, though BMI values did not significantly differ between the two BMD categories. This interesting observation is in accordance with a previous study and should be investigated further, especially looking for the role of fatsoluble vitamins, such as vitamins A, D and K, in a potential association between adiposity and BMD. In our study BMI between $19-25 \mathrm{~kg} / \mathrm{m}^{2}$ were $52 \%, 25-29.9 \mathrm{~kg} / \mathrm{m}^{2}$ were $24 \%, 17 \%$ were $>30 \mathrm{~kg} / \mathrm{m}^{2}$ and only $8 \%$ were $<19 \mathrm{~kg} / \mathrm{m}^{2}$. BMD value of L1-4 and Total femur showed positive correlation with BMI $(r=+.285$ and $\mathrm{p}<.05)$ and it was highly significant which also similar with above studies.

Another study done in 813 postmenopausal Mexican-Mestizo women in De laet,C.Kanis et al (2005). ${ }^{12}$ Height and weight were used to calculate BMI, whereas BMD in the lumbar spine (LS) and total hip (TH) were measured by dual-energy x-ray absorptiometry. They used ANOVA to examine the relationship between BMI and BMDs of the LS, TH, and femoral neck (FN), adjusting for confounding factors. The higher the BMI, the higher was the BMD at the LS, TH, and FN. The greatest differences in size variations in BMD at these three sites were observed when comparing women with normal BMI versus women with grade 3 obesity. They concluded that higher BMI is associated significantly and positively with a higher BMD at the LS, TH, and FN which was also suggested with our study.

Tarek Fawz, Jayakumary muttappallymylai et al $(2011)^{13}$ found that low weight and BMI predict osteoporosis and are associated with increased fracture risk in younger women. The negative impact of low body weight on Body Mass Index (BMI) is a good indicator for measurements of Bone Mineral Density (BMD). This study was conducted to assess the association between BMI and status of BMD among 101 individuals who underwent DualEnergy X-ray Absorptiometry (DEXA) scan. Thirty nine subjects had normal and 62 had low bone mineral density. Low BMD was recorded in $59.1 \%$ of females and $76.9 \%$ of males. Association between advancing age and lower BMI is an important risk factor in the 
occurrence of low BMD. In our study no significant negative correlation was found between advancing age and BMI but had positive significant correlation between $\mathrm{BMI}$ and BMD value ; $r=+0.285$ (L1-4) and +.350 (total Femur) $\mathrm{P}=<.01$.

BMD at the femoral neck and BMI observed was highly positive in a cross-sectional study conducted among postmenopausal women by Steinschneider et al (2003) ${ }^{11}$. The findings suggest that the increased BMD commonly reported in overweight women may result from soft tissue interference with BMD determination. Similar studies by Felson et al ${ }^{14}$ Nguyen et al ${ }^{15}$ and Baheiraei et al. ${ }^{16}$ also reported the consistent finding that lower BMI was associated with lower BMD.

In our study mean T- scores L1-4 was -2.2 and Total femur was -1.4. Among 100 patients 35 patient had normal bone mass, 44 patient had low bone mineral density and 21 patients had osteoporosis. There was positive correlation between weight and T-score of spine and Femur $(\mathrm{r}=+.482$ and $\mathrm{r}=+.513, \mathrm{P}<.01)$ which was statistically highly significant. So, it was concluded that more weight more bone mineral density. BMI presented significant positive relationships with $\mathrm{T}$ score and BMD of spine and $\mathrm{BMD}, \mathrm{T}$ score, and $\mathrm{Z}$ score of femur, whereas age, the number of years since menopause. The number of years since menopause had a positive relationship with reductions in $Z$ score and BMD of femur. In addition, significant positive relationships were observed between the number of reproductive years and $\mathrm{T}$ score and BMD of spine. They concluded bone loss in postmenopausal women is intensified with increases in age, the number of years since menopause, and the serum level of testosterone, whereas BMI has a boneprotective effect. Our study showed same results but hormonal study not done. Further studies should be recommended relationship between hormonal level with BMD value.

The present study revealed a positive and statistically significant correlation between BMI and BMD of both spine and Hip. BMD value is more, when BMI is more. The study also suggests that weight, age, duration of menopause has an effect on BMD. Further studies are required to investigate the effect of other factors like exposure to sunlight, calcium intake, and other habits like smoking, diet, and so forth.

\section{Conclusion:}

The present study results revealed a significant positive correlation between BMI and BMD. It can be concluded that in postmenopausal women as the BMI decreases bone mineral density also decreases. So, adequate weight and BMI necessary for the prevention of osteoporosis.

\section{Limitations:}

This present study was conducted in one tertiary hospital with a small sample size hence it may not reflect whole population.

\section{Recommendation:}

Further studies are required in a large nationally representative sample. Dietary habit and calcium intake should be evaluated for further study.

\section{References:}

1. Jeffcoate's principles of gynaecology, eight edition, $2014, \mathrm{P}-82$.

2. BBS- Bangladesh Bureau of statistics. Report of life expectancy -2017 .

3. Lloyd JT, Alley DE. Body mass index is positively associated with bone mineral density in us older adults. Arch Osteoporos. 2014;9:175.

4. D. Keith Edmonds, Dewhurt's textbook of obstetrics and gynaecology, 7 th edition, 2007; 479.

5. Burge R, Dawson-Hughes B, Solomon DH, incidence $\&$ economic burden of osteoporosis related fractures in USA.2005-2025.J. Bone miner Res,2007;22: 465-75.

6. Giles Jt, Ling SM, Ferrucci L,Muller ,D, Abnormal body compositionin older Rheumatoid arthritis patient associated with disesse, Arthitis Rheum 2008:19:

7. World Health organization (1994) . Assesesment of fracture risk and it's application to screening for post menopausal osteoporosis. Technical report 
series.Geneva : WHO p. 843. Operator's guide Norland XR-46 a cooper surgical Company (2003).

8. Khan AA, Syed Z (2004), Bone densitometry in premenopausal women, J Clin Densitom 17 (1): 85.

9. Masazumi, A, 2002. The effects of Body mass index on age at menopause. Inter J obs July, 26, 961-968.

10. Paulo G. Pederia, Marcelo,VeraL, arthritis Reaserch. com, 2011.p-131.

11. M. Steinschneider, P.Hagag, M. J.Rapoport, Discordant effect of BMI on BMD and speed of sound, BMC Musculoskeletal Disorders,2003 vol.4p-1-6.

12. De Laet C, Kanis J.A. Body mass index as a predictor of fracture risk: osteoporosis, Int. 2005;16:13301338.
13. Tarek Fawzy.Jayakumary muttappallymylil, J. of osteoporosis, vol 2011,p-4.

14. D.T. Felson, effects of weight and BMI on BMD in men and women: Framingham study, J. bone mine. Res, 2000,vol-8,no52 p-567-573.

15. T.V.Nguyen, Osteoporosis in elderly men and women; effects of dietary calcium, physical activity and BMI, J.bone mine res, 2000,vol-15,no2,p-322-331.

16. A. Baheiraei, N.A. Pocock, Bone mineral Density, BMI and cigarette smoking among Iranian women implications for prevention,BMC,2005, vol.6, article 34 